\title{
THE GASTROCNEMIUS MYOCUTANEOUS FLAP USED AS A COVER FOR THE EXPOSED KNEE PROSTHESIS
}

\author{
ROY SANDERS, TREVOR O'NEILL \\ From Mount Vernon Hospital and the Royal National Orthopaedic Hospital, Stanmore
}

\begin{abstract}
Exposure of the prosthesis is one of the problems encountered after arthroplasty of the knee. Eight patients with nine exposed prostheses have been treated by using a gastrocnemius myocutaneous flap to provide cover. This preserved the prosthesis and closed the joint in six of the eight patients. The factors leading to breakdown of the wound are discussed and the anatomy of the gastrocnemius myocutaneous flap and the operative procedure are described.
\end{abstract}

A greater understanding of the blood supply to the skin in recent years has led to the wider use of myocutaneous flaps on the lower limb. By virtue of its range of rotation the gastrocnemius myocutaneous flap, as described by McCraw, Dibbell and Carraway (1977) is a suitable flap for providing skin cover for the upper tibia, the knee and the lower femur.

Hip replacement is one of the most commonly performed arthroplasties and is relatively free from complications. Waugh (1978) emphasised that this had led patients to expect the same of knee arthroplasties, which are performed to the extent of 25000 per year (Black 1978). Unfortunately, knee arthroplasties are often more complicated (Arden 1975; Phillips and Taylor 1975; Attenborough 1978; Lettin et al. 1978) mainly due to serious problems of poor skin cover and subsequent infection of the exposed prosthesis caused by the subcutaneous position of the joint. Established infection in a joint may necessitate removal of the prosthesis and arthrodesis. However, attempts at arthrodesis after removal of the prosthesis are reported by Broderson et al. (1979) to be successful in only 56 to 81 per cent of patients in two groups of failed arthroplasties. The reason for failure was severe bone loss, persistent sepsis and loss of bone apposition after manipulation. A failed arthrodesis may necessitate amputation at or above the level of the joint.

\section{CLINICAL MATERIAL AND METHODS}

The factors which affect healing and predispose to breakdown of the wound may also have a bearing on the reconstruction of the defect. All our patients were over 50 years old and were therefore in the age group which may be expected to heal least successfully after arthroplasty. The age range was 50 to 88 years (average 64 years); all were fit and none were bed-ridden. There were seven women and one man in the series. Many of these patients requiring arthroplasty had had previous operations on the knee: osteotomy (three knees); synovectomy (one); patellectomy (two); and arthroplasty (three). A previous axial scar parallel to the incision used for joint replacements produces a bipedicle flap or an island of skin surrounded by scar, both of which may become necrotic (Fig. 1). Poor healing of the wound may result in separation of the edges when sutures are removed and resuture may be impossible or ineffective.

Four of our patients suffered from rheumatoid arthritis and had been taking steroids for long periods. In these patients the dermis and joint capsule were not strong, they had poor resistance to tension, and there was greater chance of the wound breaking down. Anaemia also contributes to the breakdown of the wound but is less important than other factors such as age, rheumatoid arthritis, recent operation and chronic infection.

When the wound does break down and the prosthesis becomes exposed, the time taken waiting for possible closure by granulation greatly increases the risk of infection and therefore cannot be justified.

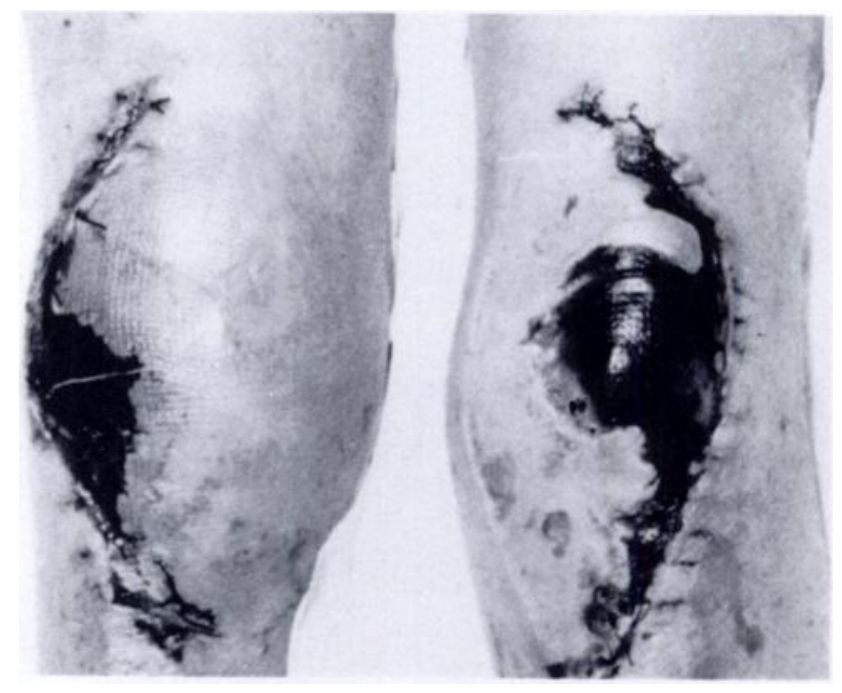

Fig. 1

Skin necrosis over the joints in a patient after bilateral arthroplasty. The greater loss has occurred on the left knee where the incision has been made parallel to an old scar.

R. Sanders, FRCS, Consultant Plastic Surgeon $\}$ Mount Vernon Hospital, Northwood, Middlesex, England.
T. O'Neill, FRCS, Registrar in Plastic Surgery

Requests for reprints should be sent to Mr R. Sanders.

(1) 1981 British Editorial Society of Bone and Joint Surgery 0301-620X/81/3076-0383 $\$ 2.00$ 
Since free skin grafts will not produce closure over an exposed joint, flap cover is necessary. Previous incisions, and the situation and the size of the defect, will usually preclude the use of local flaps. Adjacent to the knee, a compound skin and muscle flap is available. Even in the aged, the gastrocnemius myocutaneous flap is long, mobile, versatile and safe. It allows closure of large defects in more than one layer and provides good cover to permit stable, long-term healing.

Gastrocnemius myocutaneous flap. The gastrocnemius myocutaneous flap has been described comprehensively by McCraw, Dibbell and Carraway (1977), McCraw, Fishman and Sharzer (1978) and Feldman, Cohen and May (1978). Previous work has shown that areas of skin overlying muscle gain much of their blood supply through perforating vessels from the muscle. For this reason, if the muscle is raised with a skin flap then a longer more mobile flap of skin can be raised. It is necessary that the muscle receives its blood supply from a large dominant blood vessel in order that the flap may be pivoted about this point. The gastrocnemius muscle receives most of its blood supply at the level of the knee and has no significant segmental supply. The medial and the lateral muscle bellies receive independent supplies which enable them to be raised separately. In this series both the medial and the lateral flaps have been used (Fig. 2).

Medial flap. The medial boundary of this flap is the border of the tibia following the long saphenous vein, and the flap itself extends to the midline posteriorly. The distal limit of the flap may not extend beyond a point five centimetres above the medial malleolus and at this point the muscle is tendinous.

Lateral flap. The lateral flap is narrower and shorter than the medial since the lateral belly of the muscle is smaller. The lateral boundary of this flap is the fibula; the medial boundary extends to the midline posteriorly; and the distal limit should not extend beyond a point 10 centimetres above the lateral malleolus (Fig. 3). The lateral flap will not move as readily as the medial flap due to restriction by the lateral popliteal nerve where it passes over the pedicle (Fig. 4).

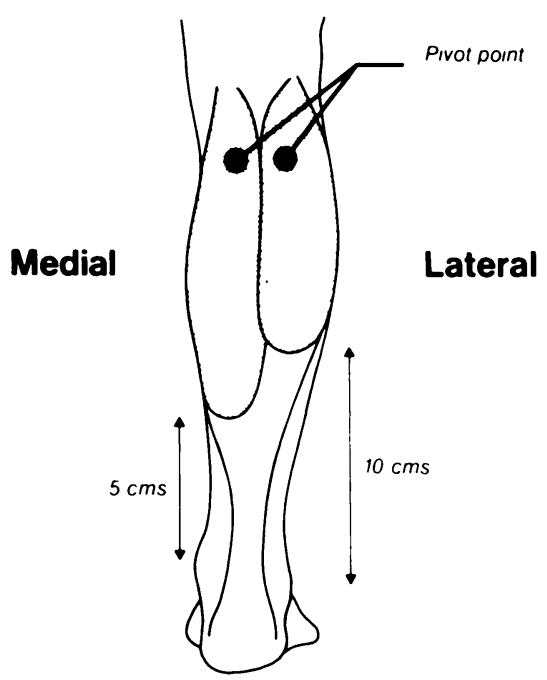

Fig. 2

Diagram of the gastrocnemius myocutaneous flaps. There is a difference in length between the lateral and the medial flap.

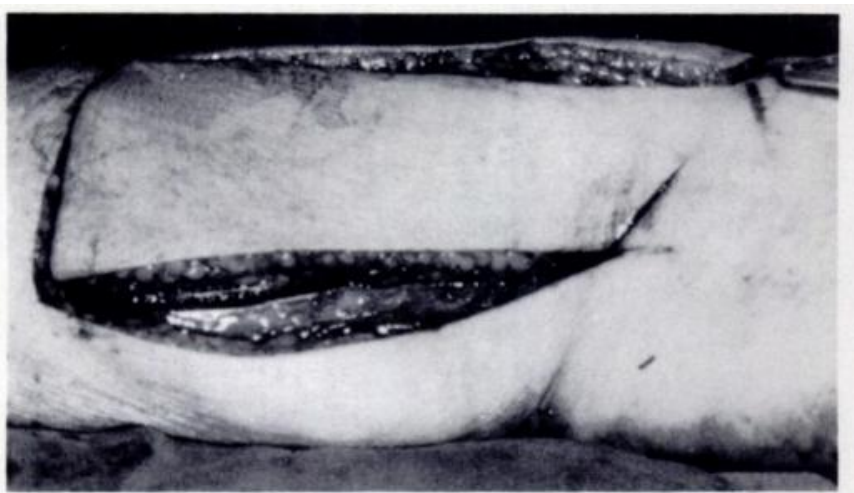

Fig. 3

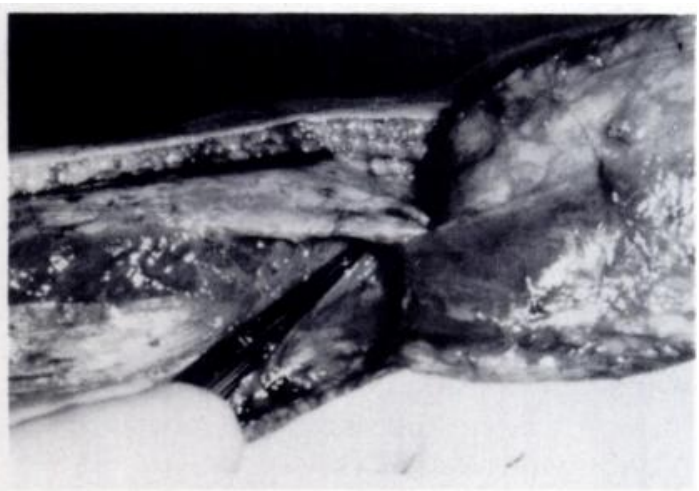

Fig. 4

Figure 3-A lateral gastrocnemius myocutaneous flap at the stage of skin incision seen from the back of the leg. Figure 4-A lateral flap has been lifted to the right. The forceps point to the lateral popliteal nerve which impedes the mobility of the pedicle.

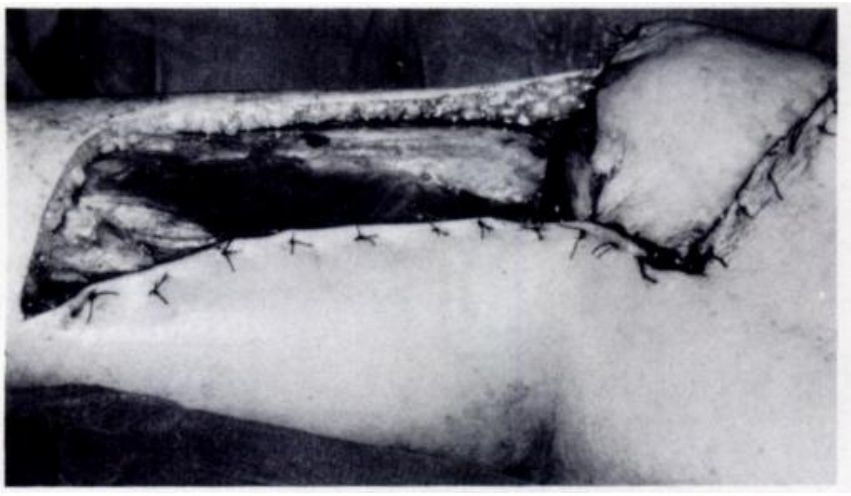

Fig. 5

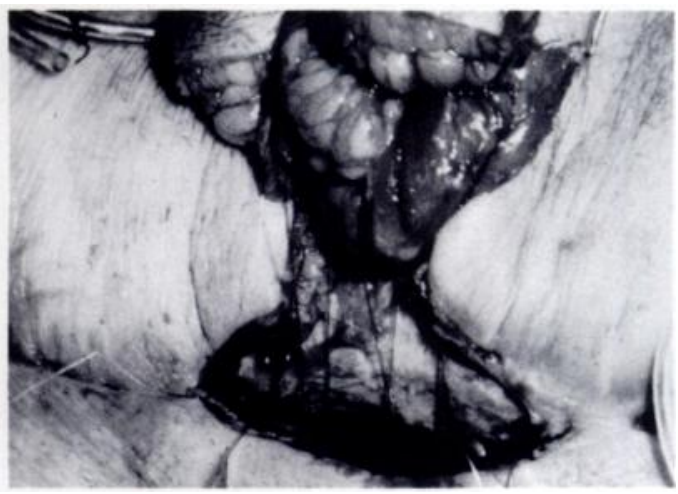

Fig. 6

Figure 5-The flap rotated and sutured in place. The skin bridge at its base has been divided to eliminate the dog ear. The size of the donor site has been reduced by tacking sutures and will be covered by a split skin graft. Figure 6-The free end of the flap being placed into position near the defect. Deep tension sutures in the muscle have been placed underneath the skin edge. This allows the muscle to be used as a reinforcing layer. 
Choice of flap. The decision whether to use a medial or lateral flap is governed by the position of the defect. The lateral flap is easier to raise because it is more accessible, but it is less mobile and shorter. The medial flap, although technically more difficult to raise, is mobile and long enough to close defects on the lateral side of the knee and is therefore preferable.

Operative technique. The posterior midline of the calf should be marked in the ward before operation since it will be distorted by the position of the patient on the table. The flaps are raised by incising the posterior midline and locating the plane of dissection between the two heads of the gastrocnemius and then between gastrocnemius and soleus. The tendinous portion of the muscle is divided distally and at this stage it is useful to tack the muscle to the skin to prevent shearing and disruption of the delicate perforating vessels.

The anterior border is then incised and the flap raised. The skin at the base of the flap can be completely incised and the flap used as an island, giving greater mobility and avoiding production of a "dog ear" (Fig. 5). Once the flap is raised the defect on the knee can be delineated and the skin excised. The defect should be made to fit the flap rather than the flap to fit the defect. The skin underlying the pedicle can be safely excised since the flap is predictable and efficient. The margin of the wound should be mobilised locally to allow tension-free approximation to the margin of the flap.

The wound is closed in three layers. The muscle may be sutured beyond the margin of the wound to allow double breasting of the layers which reinforces the closure (Fig. 6). Subcutaneous sutures approximate the rather bulky flap to the mobilised edges of the wound and suture of the skin without tension completes the procedure. The donor site is then covered with a split skin graft.

\section{RESULTS}

In five patients the original pathology was rheumatoid arthritis and in the remaining three it was osteoarthritis. All eight patients had had previous operations on the knee and three had undergone a repeat arthroplasty. Five of the myocutaneous flaps were based on the medial head of the gastrocnemius and four on the lateral.

The time from exposure of the prosthesis to closure varied from 24 hours to nine months. Two patients, one of whom had an exposure for only 24 hours, suffered late infection eight months after the closure operation. The infection was controlled by long-term antibiotics and the arthroplasty was conserved. The patient who was

Table I. The results for eight patients

\begin{tabular}{|c|c|c|c|l|l|}
\hline Patient & Age & Sex & $\begin{array}{c}\text { Time before } \\
\text { closure }\end{array}$ & $\begin{array}{c}\text { Type of } \\
\text { fap used }\end{array}$ & \multicolumn{1}{|c|}{ Result } \\
\hline 1 & 59 & M & 24 hours & Medial & Late infection \\
2 & 65 & F & 3 months & Medial & Healed \\
3 & 88 & F & 1 month & Lateral & Healed \\
4 & 68 & F & 5 months & Medial & Late infection \\
5 & 56 & F & 4 months & Lateral ${ }^{*}$ & Unhealed \\
6 & 50 & F & 3 months & Medial & Arthrodesis \\
7 & 68 & F & 9 months & Medial & Healed \\
8 & 63 & F & 2 months & Lateral & Healed \\
\hline
\end{tabular}

* Bilateral unhealed for nine months, healed uneventfully after closure with a medial myocutaneous flap and remained free from complications. There. seems to be no correlation between the time before closure and the success rate in this small series, although obviously rapid closure is preferable.

Despite delays in skin closure of between one day and nine months, bacterial culture produced skin commensals only, in three patients, Staphylococcus albus in three and no growth in the remaining two. Late clinical infection developed in two of the seven successful closures made using a myocutaneous flap (Table I).

We have previously encountered problems with the gastrocnemius myocutaneous flap in patients who had been treated for other conditions and who had suffered previous deep vein thrombosis. These usually resulted in loss of the margin of the flap. In this series part of one

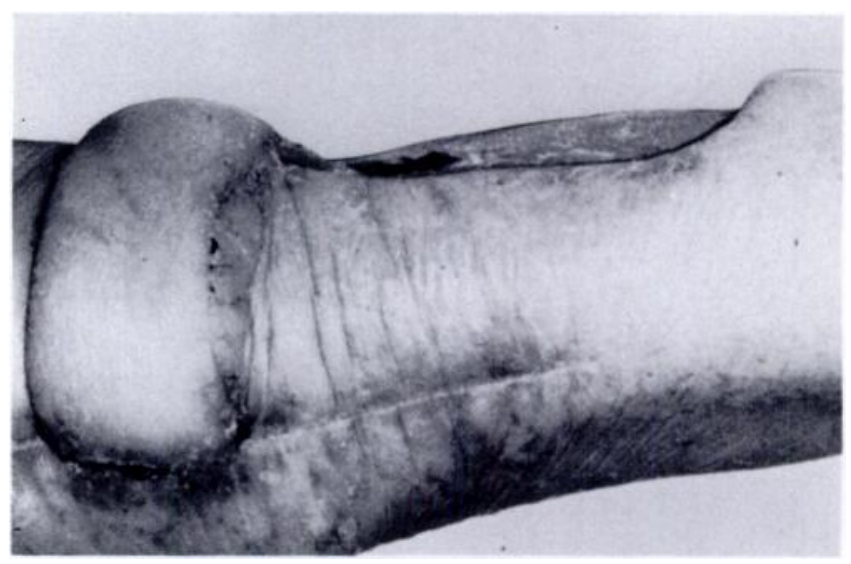

Fig. 7

The flap is seen at three weeks when the patient is walking and free of pain. The donor site defect can be seen.

flap was lost probably for the same reasons, since the patient was known to have had a deep vein thrombosis in the past. Closure has not yet been achieved in this patient. A second patient developed infection of the arthroplasty immediately after operation and the prosthesis was removed. All six patients who healed primarily were discharged from hospital fully healed. three weeks after closure (Fig.7). They were able to walk and were free from pain. There was no functional deficit resulting from the flap in this series and the cosmetic defect seems fully justified in view of the conservation of the arthroplasty and the leg.

\section{DISCUSSION}

In knee arthroplasties exposure of the almost subcutaneous prosthesis occurs in the event of breakdown of the wound. Such breakdown is more likely in the elderly, or those on steroid therapy. Badly planned skin incisions are a common cause of wound dehiscence. A 
second incision on the front of the knee parallel to or joining a previous scar is likely to devascularise the skin, resulting in necrosis of the skin or failure to heal. Previously, exposure and subsequent infection of the prosthesis necessitated its removal and attempted arthrodesis. It is now possible to avoid this and to preserve the prosthesis and the function of the knee in the majority of patients suffering this complication.

The joint and prosthesis are covered with a gastrocnemius flap with a fresh blood supply which may help combat the infection present, as well as providing a stable and reliable skin cover even in the elderly. If there is doubt concerning the viability of the flap after it has been raised the fluorescein test, using one gram of five per cent solution injected intravenously, is a reliable method of ascertaining it (McCraw, Myers and Shanklin 1977): in this test the flap is viewed with the aid of ultraviolet light 30 minutes after the injection and any parts that fluoresce inadequately are of dubious viability. The flap can then either be put back into its bed and the operation used as a form of "delay" or if there is excess flap available, the non-viable portion can be excised.

Selection of the flap to be used is governed by the position of the defect, but in many cases lateral defects can be closed by the use of the medial flap which is a longer, more robust, and more mobile flap. Deep vein thrombosis may be the cause of impaired viability of similar flaps in other patients outside our series, and it should therefore be considered as a possible hazard.

The gastrocnemius flaps offer a salvage procedure when a prosthesis becomes exposed, and should be contemplated before proceeding to removal of the prosthesis and arthrodesis. In patients with previous scars over the knee and in whom arthroplasty is intended, the gastrocnemius flaps may be used at the time of insertion of the prosthesis to obviate the development of breakdown of the wound. It is a single-stage procedure providing safe, adequate cover and permitting early mobilisation.

We would like to thank Mr Brian Morgan for allowing us to include two of his patients in the series; Mr D. H. Harrison who performed one of the procedures, and Mr R. Coombes who presented this work on our behalf at the Canterbury Meeting of the British Orthopaedic Association in April 1980. Our thanks also go to Mr R. Blake and the staff of the photographic department of Mount Vernon Hospital, and to Mrs C. Wynne for secretarial assistance.

\section{REFERENCES}

Arden GP. Total replacement of the knee. J Bone Joint Surg [Br] 1975;57-B:119-20.

Attenborough CG. The Attenborough total knee replacement. J Bone Joint Surg [Br] 1978;60-B:320-6.

Black J. The future of polyethylene. J Bone Joint Surg [Br] 1978;60-B:303-6.

Broderson MP, Fitzgerald RH, Peterson LFA, Coventry MB, Bryan RS. Arthrodesis of the knee following failed total knee arthroplasty. J Bone Joint Surg $[A m]$ 1979;61-A:181-5.

Feldman JJ, Cohen BE, May JW Jr. The medial gastrocnemius myocutaneous flap. Plast Reconst Surg 1978;61:531-9.

Lettin AWF, Deliss LJ, Blackburne JS, Scales JT. The Stanmore hinged knee arthroplasty. J Bone Joint Surg [Br] 1978;60-B:327-32.

McCraw JB, Dibbell DG, Carraway JH. Clinical definition of independent myocutaneous vascular territories. Plast Reconst Surg 1977;60:341-52.

McCraw JB, Myers B, Shanklin KD. The value of fluorescein in predicting the viability of arterialized flaps. Plast Reconst Surg 1977;60:710-19.

McCraw JB, Fishman JH, Sharzer LA. The versatile gastrocnemius myocutaneous flap. Plast Reconst Surg 1978;62:15-23.

Phillips H, Taylor JG. The Walldius hinge arthroplasty. J Bone Joint Surg [Br] 1975;57-B:59-62.

Waugh W. Knee replacement 1978. J Bone Joint Surg [Br] 1978;60-B:301-3. 